\title{
Self-collection for high-risk HPV detection in Brazilian women using the careHPV ${ }^{\mathrm{TM}}$ test
}

\author{
Adriana Tarlá Lorenzi ${ }^{\text {a,* }}$, José Humberto T.G. Fregnani ${ }^{b}$, Júlio César Possati-Resende ${ }^{c}$, \\ Cristovam Scapulatempo Neto ${ }^{\mathrm{d}}$, Luisa Lina Villa ${ }^{\mathrm{e}, \mathrm{f}}$, Adhemar Longatto-Filho ${ }^{\mathrm{b}, \mathrm{g}, \mathrm{h}, \mathrm{i}, *}$ \\ ${ }^{a}$ Molecular Oncology Center, Barretos Cancer Hospital, Barretos, São Paulo, Brazil \\ b Center for the Researcher Support, Barretos Cancer Hospital, Barretos, São Paulo, Brazil \\ c Cancer Prevention Department, Barretos Cancer Hospital, Barretos, São Paulo, Brazil \\ d Pathology Department, Barretos Cancer Hospital, Barretos, São Paulo, Brazil \\ e Santa Casa de São Paulo Medical School, FCMSCSP, São Paulo, Brazil \\ ${ }^{\mathrm{f}}$ Faculty of Medicine, Department of Radiology and Basic Oncology, São Paulo University, FMUSP, São Paulo, Brazil \\ ${ }^{g}$ Laboratory of Medical Investigation (LIM) 15, Faculty of Medicine, São Paulo University, FMUSP, São Paulo, Brazil \\ ${ }^{\mathrm{h}}$ Life and Health Sciences Research Institute, ICVS, School of Health Sciences, Minho University, Braga, Portugal \\ i ICVS/3B's-PT Government Associate Laboratory, Braga/Guimarães, Portugal
}

\section{H I G H L I G H T S}

- High-risk HPV was tested in careHPVTM methodology.

- HPV test self-sampled material is useful for cervical cancer screening.

\section{A R T I C L E I N F O}

\section{Article history:}

Received 12 June 2013

Accepted 16 July 2013

Available online xxxx

\section{Keywords:}

Human papillomavirus

Cervical cancer

Self-sampling

Molecular test

Screening

\section{A B S T R A C T}

Objective. Cervical cancer is the second most common cancer among Brazilian women. High-risk human papillomavirus (hr-HPV) persistence is the primary cause of cervical neoplasia. Early detection of hr-HPV is important for identifying women at risk for developing cervical lesions. Approximately $85 \%$ of new cases of cervical cancer worldwide and $50 \%$ of the total cervical cancer deaths occurred in developing countries. Here, a new methodology to support a cervical cancer screening program was evaluated in women from various Brazilian regions.

Methods. Two thousand women aged 18-77 years were enrolled in an opportunistic cervical cancer screening program and were randomized into self-vaginal or health professional-guided cervical sampling groups. The Qiagen careHPVтм test was performed on all samples. Pap tests were performed on all women using liquid-based cytology.

Results. Positive hr-HPV results were obtained in 12.3\% (245/2000) of women; similar rates were observed in self- or health professional-collected samples. Eighty-nine percent (1719/2000) of cervical cytologies classified as normal were negative to hr-HPV. Among the cytological samples, 36.6\% classified as ASC-US + were positive to hr-HPV, 78.8\% were LSIL and 75.0\% were HSIL.

Conclusions. Self-sampled and health professional-sampled vaginal/cervical specimens did not differ in their rates of detection of hr-HPV. Therefore, HPV DNA testing in self-sampled vaginal cells is an alternative to primary screening in low-resource settings.

(C) 2013 Elsevier Inc. All rights reserved.

\section{Introduction}

Cervical cancer (CC) is a well-known disease worldwide and remains a critical public health issue in developing countries that

\footnotetext{
* Corresponding author at: Laboratory of Medical Investigation (LIM) 15, Faculty of Medicine, São Paulo University, São Paulo 1246-903, Brazil. Fax: + 551130617413.

E-mail addresses: adriana.lorenzi@gmail.com (A.T. Lorenzi), mdfregnani@terra.com.br (J.H.T.G. Fregnani), julio.possati@uol.com.br (J.C. Possati-Resende),

cristovamscapula@uol.com.br (C.S. Neto), luisapvilla@gmail.com (L.L. Villa),

longatto16@hotmail.com (A. Longatto-Filho).
}

lack appropriate screening programs. Consequently, 85\% of cases and $50 \%$ of deaths due to CC occur in developing countries, particularly in the poorest regions. Most Brazilian regions have dramatic indexes of mortality: CC is the second most common cancer among Brazilian women, with mortality rates estimated to be more than 10,000 deaths annually and more than 17,000 new cases occurring each year $[1,2]$.

Human papillomavirus (HPV) infections are prevalent worldwide. Persistent hr-HPV infection is the single main risk factor for the development of premalignant and malignant cervical lesions [3]. HPV causes virtually all cases of cervical cancer [4]. Cervical cancer screening 
programs are tailored to optimize the identification of precursor lesions of cervical cancer, thereby contributing to reduction of the associated mortality. Cervical cancer develops over a long period of time (approximately 20 years from the initial HPV infection to the onset of invasive cancer), which allows for preventive actions. [5]. A combination of HPV testing with liquid-based cytology screening has been demonstrated to improve the algorithm to protect women against CC. HPV DNA testing is considered to be the most efficient tool to screen women at risk for cervical diseases because of the higher sensitivity in comparison to the cytology examination [6-8]. This superior sensitivity prevents the high rates of false-negative results usually associated with cytological examination. As the cytology demands very qualified skill and stringent internal quality control system, HPV test could be an alternative to optimize the cervical screening programs in countries or regions where the cytology test is not feasible to be implemented.

Recently, a new molecular HPV DNA test (careHPVTM) was developed to detect 14 of the main hr-HPV strains $(16,18,31,33,34,39,45,51,52$, $56,58,59,66$ and 68 ) in low-resource or technically poor settings. The careHPV ${ }^{\mathrm{TM}}$ system does not require electricity or flowing water and provides results for up to 90 samples in a total of $4 \mathrm{~h}$. The test is portable and can be performed under suboptimal laboratory conditions and in very small spaces. Moreover, the test is a user-friendly and reproducible system that encompasses a few easy steps [9]. In comparison with the performance of the conventional Pap test, HPV DNA testing is more sensitive, practical and analytically objective $[5,10,11]$. Despite the lower positive predictive value of self-sampled HPV DNA testing compared with cytology, a recent study [12] demonstrated that notable HPV results improved the detection of CIN2 + in developing countries, particularly in settings with poor human and technology resources. The sensitivity of HPV DNA testing is fundamental for women with limited access to traditional screening. We anticipated that this HPV test could be an excellent option for primary screening in many regions of Brazil. The objective of this study was compare the careHPV ${ }^{\mathrm{TM}}$ test results from self-sampling and health professional collection specimens.

\section{Methods}

\section{Study design}

This study was performed between March and December 2012 at the ambulatory wing of the Cancer Prevention department of Barretos Cancer Hospital (BCH), Barretos, São Paulo, Brazil. Women were randomized into two groups: vaginal self-sampling vs. cervical health professional sampling. Sequentially numbered envelopes were prepared by a statistics department member of this institution $(\mathrm{BCH})$ to guarantee adequate randomization. None of the professionals who implemented the randomization or done the sample collection knew the content of the envelops (if the samples were self-sampled or collected by professionals health) or the results of the other tests. This study was registered in Clinical Trials (no. NCT01539668).

\section{Ethics}

All women received a patient information sheet explaining the study and provided written consent. The eligible women were invited and number registered to participate. Approvals were obtained from the BCH Research Ethic Committee.

\section{Subjects and specimen collection}

The study enrolled 2000 volunteer women in an opportunistic screening at BCH. Eligible women were older than 18 years. Participants were informed about the HPV test, CC, the importance of early detection and cervical cancer prevention and the study objectives. After signing the informed consent form, each woman picked up an envelope in the number sequence that provided information about the study group. If she was sorted for self-sampling, a well-trained professional instructed the woman with an illustrative cartoon concerning how to take the vaginal sample without professional intervention; alternatively, the study nurse collected a standard cervical sample if the woman was referred to the professional sampling group.

\section{Sample collection}

The first sample collected was for hr-HPV diagnosis using the careHPV TM test. A conical-shaped brush (Qiagen, Gaithersburg, MD, USA) was used to take the vaginal specimen: the woman inserted the brush head into the vagina up to $10 \mathrm{~cm}$, rotated it (clockwise) three times before removal and placed the brush immediately in a careHPV TM medium tube (QIAGEN, USA), which was delivered to and capped by the study personnel. The health professional interviewed the women in both groups to complete a structured questionnaire with socio-demographic information.

\section{Pap test collection and preparation}

A second specimen was collected for cervical cytology by a welltrained professional using a Rover's cytobrush with a removable brush head (Rover's, Holland). An endocervical and an ectocervical canal sample were placed immediately in SurePath $®$ (Becton Dickinson, USA). Specimens were sent to the BCH Pathology Department, Barretos, São Paulo, Brazil, and then were analyzed and classified using the BD FocalPoint ${ }^{\mathrm{TM}}$ Slide Profiler. The specimens were reviewed by a cytotechnician, and altered cases (ASC-US +) were revised by a cytopathologist.

\section{HPV DNA test}

HPV DNA analysis was performed at the BCH Oncology Molecular Center, Barretos, São Paulo, Brazil, using the careHPVTM test, which comprises an in vitro nucleic acid hybridization assay with signal amplification using microplate chemiluminescence for the qualitative detection of 14-h HPV types $(16,18,31,33,35,39,45,51,52,56,58,59,66$ and $68)$ in cervical specimens [13].

Women were informed by letter about their results if they were HPV negative or were recalled to follow up if they were HPV positive. Women who tested positive for hr-HPV and/or with cytology ASC-H + were referred for colposcopy and/or biopsy (if necessary).

\section{Statistical analysis}

The sample size calculation was based on the principle of an equivalence clinical trial, in which the new intervention and the standard one are equally effective. The prevalence rate of HPV infection was supposed to be around $10-15 \%$ in a low-risk population. Considering an alpha error of $5 \%$, beta error of $10 \%$ and clinical acceptable margin of $5 \%$ between self-sampling and professional sampled groups, the sample size has been estimated from 756 to 1071 subjects in each arm [14].

Statistical analysis was accomplished using the computer software IBM ${ }^{\circledR}$ SPSS ${ }^{\circledR}$ Statistics (Statistical Package for Social Sciences) version 20.1 for Windows (IBM Corporation, Route 100, Somers, NY 10589). Chi-squared $\left(\chi^{2}\right)$ test was used to compare frequencies among groups. Student's $t$-test was used to compare means between groups, and Fisher's exact test was used when the $\chi^{2}$ test was not sufficient for group comparison. In all tests, $p$ values less than 0.05 were considered to be statistically significant.

\section{Results}

A total of 2000 women aged 18-76 years were tested for hr-HPV using the careHPV ${ }^{\mathrm{TM}}$ test by either self-sampling or sample collection by a health professional. Two hundred forty-five specimens (12.3\%) 
Table 1

Comparison between the careHPV ${ }^{\mathrm{TM}}$ test, collection group and the valid socio-demographic women questionnaires.

\begin{tabular}{|c|c|c|c|c|c|}
\hline & \multicolumn{2}{|c|}{ careHPV'м test } & \multicolumn{3}{|l|}{ Source } \\
\hline & Positive & $p$ value & Self-sampling & Health professional & $p$ value \\
\hline \multicolumn{6}{|c|}{ Age (years) } \\
\hline $18-29$ & $28.2 \%(66)$ & $<0.0001$ & $55.1 \%(129)$ & $44.9 \%(105)$ & 0.051 \\
\hline $30-39$ & $17.3 \%(60)$ & & $53.6 \%(186)$ & $46.4 \%(161)$ & \\
\hline$\geq 40$ & $8.4 \%(119)$ & & $48.3 \%(685)$ & $51.7 \%(734)$ & \\
\hline \multicolumn{6}{|l|}{ Parity } \\
\hline None & $23.6 \%(66)$ & $<0.0001$ & $52.5 \%(147)$ & $47.5 \%(133)$ & 0.645 \\
\hline $1-2$ & $10.9 \%(113)$ & & 49.6\% (513) & $50.4 \%(521)$ & \\
\hline$>2$ & $9.5 \%(64)$ & & $49.3 \%(332)$ & $50.7 \%(341)$ & \\
\hline \multicolumn{6}{|c|}{ Sexual partners } \\
\hline 1 & $8,5 \%(98)$ & $<0.0001$ & $50.3 \%(577)$ & $49.7 \%(571)$ & 0.800 \\
\hline $2-3$ & $16.9 \%(81)$ & & $51.3 \%(245)$ & $48.7(233)$ & \\
\hline$>3$ & $19.1 \%(53)$ & & $48.7 \%(135)$ & $51.3 \%(142)$ & \\
\hline \multicolumn{6}{|c|}{ Tobacco use } \\
\hline Yes & $13.4 \%(31)$ & 0.587 & $49.8 \%(115)$ & $50.2 \%(116)$ & 0.970 \\
\hline No & $12.2 \%(213)$ & & $49.9 \%(874)$ & $50.1 \%(877)$ & \\
\hline \multicolumn{6}{|c|}{ Contraceptive use } \\
\hline Yes & $21.3 \%(69)$ & $<0.0001$ & $50.9 \%(165)$ & 49.1\% (159) & 0.708 \\
\hline No & $10.6 \%(175)$ & & $49.8 \%(820)$ & $50.2 \%(827)$ & \\
\hline
\end{tabular}

One unsatisfactory molecular test

tested hr-HPV positive; $96.4 \%$ of women who participated in this trial declared that they have at least one Pap test before the study, and most reported only one lifetime sexual partner. No differences were observed between the collection groups (self or health professional) regarding variables such as parity, number of sexual partners, contraceptive and tobacco use $\left(p>0.05 ; \chi^{2}\right.$ test) (Table 1$)$. However, when we considered the careHPV ${ }^{\mathrm{TM}}$ results, differences between the collection groups were observed concerning the variables. Eleven percent of valid questionnaires included smokers; among them, $13.4 \%$ were positive for hr-HPV infection. In the non-smoking population, $12.2 \%$ were positive for the molecular hr-HPV test. Regarding contraceptive use, $16.45 \%$ of the participants used contraceptives; among them, $21.3 \%$ were positive for hr-HPV. Among the women who did not use contraceptives, $10.6 \%$ were positive for the molecular hr-HPV test ( $p<0.0001 ; \chi^{2}$ test). Regarding parity and the number of sexual partners, statistically significant differences were also found.

A slightly higher frequency of hr-HPV positive results was observed in the self-sampling group compared with the professionally sampled group (13.5\% (135) versus $11 \%$ (110), respectively), but these results were not statistically significant ( $p=0.087$ ). The mean age was slightly increased in the professional collection group (45.6 years), whereas that in the self-sampling group was 44.5 years ( $p=0.034$; Student's $t$-test).

Considering the cytological results, $10.5 \%$ of the non-relevant alteration (NILM) samples were positive for hr-HPV in the molecular test.
In four HSIL cases, three (75\%) were hr-HPV positive. Additionally, 26 of 33 LSIL $(78.8 \%$ ) cases were positive for the careHPVTM test. Most (89.5\%; $n=1719$ ) of the women with NILM cytological results tested negative on the HPV molecular test (Table 2).

\section{Discussion}

HPV infection is a highly common sexually transmitted infection among young women, but it is mostly transitory. Conversely, the persistence of hr-HPV in women older than 40 years is associated with cervical cancer development. hr-HPV is the main cause of death by cancer among Brazilian women [5,11]. Currently, molecular HPV testing is indicated for screening women who are at risk for cervical lesion development because of its higher sensitivity, decreased subjectivity and better reproducibility compared with cytology [8-10]. Self-sampling is an important tool to test women for HPV infection, particularly in developing countries or remote areas, where it is possible to send such women kits with brushes, medium collection supplies and illustrative instructions. This option is relatively inexpensive and may benefit women who may not otherwise have access to the health system [15,16]. Independent of the HPV test used, generally, self-collected samples have similar frequencies of HPV DNA to samples collected by a health professional [17]. Our results endorse this premise because no difference was observed between collection procedures. Because careHPV ${ }^{\mathrm{TM}}$ is affordable and can be used in various settings with various collection samples, we

Table 2

Cytological results relative to careHPV ${ }^{\mathrm{TM}}$ test.

\begin{tabular}{|c|c|c|c|c|c|c|}
\hline & \multicolumn{3}{|c|}{ careHPV $^{\mathrm{TM}}$ test* } & \multicolumn{3}{|l|}{ Source* } \\
\hline & Negative & Positive & $p$ value & Self-sampling & Health professional & $p$ value \\
\hline NILM & $89.5 \%(1719)$ & $10.5 \%(201)$ & & $49.9 \%(958)$ & $50.1 \%(963)$ & \\
\hline ASC-US + & $63.4 \%(26)$ & $36.6 \%(15)$ & & $53.7 \%(22)$ & $46.3 \%(19)$ & \\
\hline LSIL & $21.2 \%(07)$ & $78.8 \%(26)$ & $<0.0001$ & $51.5 \%(17)$ & $48.5 \%(16)$ & 0.738 \\
\hline HSIL & $25.0 \%(01)$ & $75.0 \%(03)$ & & $75.0 \%(03)$ & $25.0 \%(01)$ & \\
\hline Total & $87.7 \%(1753)$ & $12.3 \%(245)$ & & $50.0 \%(1000)$ & $50.0 \%$ (999) & \\
\hline
\end{tabular}

The statistical test used to evaluate the groups was $\chi^{2}$, considering 0.05 of significance.

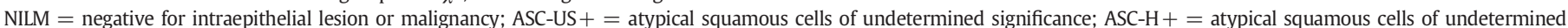

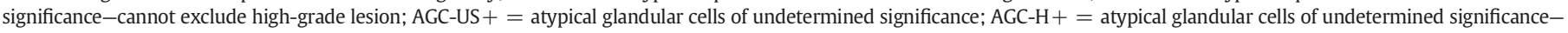
cannot exclude high-grade lesion; LSIL = low-grade squamous intraepithelial lesion; HSIL = high-grade squamous intraepithelial lesion.

*It was excluded of the statistical data analysis two samples with unsatisfactory results (one careHPV ${ }^{\mathrm{TM}}$ test and one liquid-based cytology). 
studied 2000 women subjected to careHPV ${ }^{\mathrm{TM}}$ analysis and found a positive hr-HPV reaction in up to $12 \%$ of them. The important point to emphasize is that these women live mostly in rural areas of São Paulo state and other states of the Brazilian federation, where regular medical assistance and medical facilities are scarce. However, the fraction of women we studied may not properly represent the rural population of São Paulo State. We therefore sought to test a simple and reliable HPV test in self- and non-self-collected samples to verify its potential use in rural remote areas and low-resource settings of Brazil.

Considering the socio-demographic findings, no association was found between hr-HPV positivity and tobacco use in either arm of the analysis (molecular results or collection group). Concerning parity, sexual partners and contraceptive use, no association with collection group was found. These data could be used to illustrate an adequate and homogeneous randomization system. However, these variables showed an association with hr-HPV positivity, as illustrated in Table 1 . With these data, we hypothesize that our situation involves younger women, most of whom are contraceptive users and have fewer children. In other words, newer social and professional behaviors could increase the rates of hr-HPV infection in this population.

The performance of the careHPV ${ }^{\mathrm{TM}}$ test has also been evaluated in a large study conducted in China. Its accuracy was similar to that of another molecular test (Hybrid Capture II, Qiagen)[9]. The careHPV тм test had a higher sensitivity but a lower specificity compared with liquid-based cytology and visual inspection with acetic acid (VIA) to detect CIN2 +, as described in the Chinese study. This lower specificity of the careHPV ${ }^{\mathrm{TM}}$ test can be explained by the fact that the presence of hr-HPV is not necessarily associated with a high-grade lesion [10]. However, in our study, only one HSIL cytological case was not found to be hr-HPV-positive using the molecular test. Conversely, 26 of 33 LSIL cytology (78.8\%) cases were found to be hr-HPV positive using the molecular test. This end point raises an important question regarding careHPV ${ }^{\mathrm{TM}}$ sensitivity, which differs from that observed in other studies [9].

The careHPV ${ }^{\mathrm{TM}}$ test is expected to be commercially available in developing countries in the future. The results of HPV screening for cervical cancer combined with those of the Chinese study of the new hr-HPV test indicate that HPV testing is appropriate as a primary screening approach in low-resource settings for women who are at least 30 years of age $[9,18]$. Moreover, several lines of evidence have confirmed that HPV testing is superior to cytology as a primary technical method for screening.

In conclusion, the results presented herein demonstrate that selfcollection of vaginal samples is equivalent to cervical sampling for HPV DNA detection and can be used in primary cervical cancer screening.

\section{Conflict of interest}

LLV is a consultant of Qiagen, BD and Roche for HPV DNA testing.

\section{Acknowledgments}

The authors thank the following: Cancer Prevention Department Team, Cleyton Zanardo de Oliveira and Allini Mafra of the Researcher Support Team and the Pathology Department of the Barretos Cancer Hospital. Rui Manuel Reis and André Lopes Carvalho from the Molecular Oncology Center; José Eduardo Levi from São Paulo University; Cintia B. Oliveira, Raphael Haikel Junior and Edmundo Mauad from Barretos Cancer Hospital. Luisa Lina Villa was supported by a grant from CNPq and FAPESP (INCT-HPV). Study Supported by CNPq - Process $n^{\circ} 573799 /$ 2008-3 and FAPESP $n^{\circ}$ 2008/57889-1. They also thank all volunteer women who participated of this study.

\section{References}

[1] WHO/ICO. Cancer incidence, mortality and prevalence worldwide in 2008. Lyon: World Health Organization; 2008 [8 November 2011]; 2008:[Available from: http://globocan.iarc.fr.].

[2] WHO/ICO. Human papillomavirus and related cancers in Brazil. Summary report 2010. Lyon: WHO/ICO Information Centre on HPV and Cervical Cancer; 2010 [cited 201122 December 2011]; Available from: http://globocan.iarc.fr].

[3] Schiffman M. Integration of human papillomavirus vaccination, cytology, and human papillomavirus testing. Cancer Jun 25 2007;111(3):145-53.

[4] Crawford R, Grignon AL, Kitson S, Winder DM, Ball SL, Vaughan K, et al. High prevalence of HPV in non-cervical sites of women with abnormal cervical cytology. BMC Cancer 2011;11:473.

[5] INCA. Câncer no Brasil: dados dos registros de base populacional. Brasilia: Ministerio da Saúde; 2010 [Contract No.: 10/12/2012].

[6] Cox T, Cuzick J. HPV DNA testing in cervical cancer screening: from evidence to policies. Gynecol Oncol Oct 2006;103(1):8-11.

[7] Franco EL, Cuzick J. Cervical cancer screening following prophylactic human papillomavirus vaccination. Vaccine Mar 14 2008;26(Suppl. 1):A16-23.

[8] Longatto-Filho A, Schmitt FC. Gynecological cytology: too old to be a pop star but too young to die. Diagn Cytopathol Oct 2007;35(10):672-3.

[9] Qiao YL, Sellors JW, Eder PS, Bao YP, Lim JM, Zhao FH, et al. A new HPV-DNA test for cervical-cancer screening in developing regions: a cross-sectional study of clinical accuracy in rural China. Lancet Oncol Oct 2008;9(10):929-36.

[10] Zhao FH, Lewkowitz AK, Chen F, Lin MJ, Hu SY, Zhang X, et al. Pooled analysis of a self-sampling HPV DNA test as a cervical cancer primary screening method. J Natl Cancer Inst Feb 8 2012;104(3):178-88.

[11] Ho GY, Bierman R, Beardsley L, Chang C], Burk RD. Natural history of cervicovaginal papillomavirus infection in young women. N Engl J Med Feb 12 1998;338(7):423-8.

[12] Lazcano-Ponce E, Lorincz AT, Cruz-Valdez A, Salmeron J, Uribe P, Velasco-Mondragon $\mathrm{E}$, et al. Self-collection of vaginal specimens for human papillomavirus testing in cervical cancer prevention (MARCH): a community-based randomised controlled trial. Lancet Nov 26 2011;378(9806):1868-73.

[13] Qiagen. The careHPV test training guide. Germantown: Qiagen Corporation; 2008.

[14] Zhong B. How to calculate sample size in randomized controlled trial? J Thorac Dis Dec 2009;1(1):51-4.

[15] Silva J, Ribeiro J, Sousa H, Cerqueira F, Teixeira AL, Baldaque I, et al. Oncogenic HPV types infection in adolescents and university women from north Portugal: from self-sampling to cancer prevention. J Oncol 2011;2011:953469.

[16] Ogilvie GS, Patrick DM, Schulzer M, Sellors JW, Petric M, Chambers K, et al. Diagnostic accuracy of self collected vaginal specimens for human papillomavirus compared to clinician collected human papillomavirus specimens: a meta-analysis. Sex Transm Infect Jun 2005;81(3):207-12.

[17] Baldwin S, Santos C, Mendez Brown E, Nuno T, Giuliano A, Davis J, et al. Comparison of type-specific human papillomavirus data from self and clinician directed sampling. Gynecol Oncol May 2005;97(2):612-7.

[18] Sankaranarayanan R, Nene BM, Shastri SS, Jayant K, Muwonge R, Budukh AM, et al. HPV screening for cervical cancer in rural India. N Engl J Med Apr 2 2009;360(14):1385-94 